\title{
Statistical properties of stock order books: empirical results and models
}

\author{
Jean-Philippe Bouchaud ${ }^{\dagger, *}$, Marc Mézard $^{* *, *}$, Marc Potters* $^{*}$
}

February 6, 2008

† Commissariat à l'Energie Atomique, Orme des Merisiers

91191 Gif-sur-Yvette CEDEX, France

* Science \& Finance, CFM, 109-111 rue Victor Hugo

92353 Levallois CEDEx, France

** Laboratoire de Physique Théorique et Modèles Statistiques

Université Paris Sud, Bat. 100, 91405 Orsay CeDEx, France

February 6, 2008

\begin{abstract}
We investigate several statistical properties of the order book of three liquid stocks of the Paris Bourse. The results are to a large degree independent of the stock studied. The most interesting features concern (i) the statistics of incoming limit order prices, which follows a power-law around the current price with a diverging mean; and (ii) the shape of the average order book, which can be quantitatively reproduced using a 'zero intelligence' numerical model, and qualitatively predicted using a simple approximation.
\end{abstract}

Financial markets offer an amazing source of detailed data on the collective behaviour of interacting agents. It is possible to find many reproducible patterns and even to perform experiments, which bring this atypical subject into the realm of experimental science. The situation is simple and well defined, since many agents, with all the same goal, trade the very same asset. As such, the statistical analysis of financial markets also offers an interesting testing ground not only for economic theories, but also for more ambitious theories of human activities. One may indeed wonder to what extent it is necessary to invoke human intelligence or rationality to explain the various universal statistical laws which have been recently unveiled by the systematic analysis of very large data sets.

Many statistical properties of financial markets have already been explored, and have revealed striking similarities between very different markets (different 
traded assets, different geographical zones, different epochs) [1], 3, 2]. For example, the distribution of price changes exhibits a power-law tail with an apparently universal exponent [4, 5, 6, 8]. The volatility of most assets shows random variations in time, with a correlation function which decays as a small power of the time lag, again quite universal across different markets [7].

Here, we study the statistics of the order book, which is the ultimate 'microscopic' level of description of financial markets. The order book is the list of all buy and sell 'limit' orders, with their corresponding price and volume, at a given instant of time. A limit order specifies the maximum (resp. minimum) price at which an investor is willing to buy (resp. sell) a certain number of shares (volume). At a given instant of time, all limit buy orders are below the best buy order called the bid price, while all sell orders are above the best sell order called the ask price. When a new order appears (say a buy order), it either adds to the book if it is below the ask price, or generates a trade at the ask if it is above (or equal to) the ask price (we call all these 'market orders' even if technically they could also be 'marketable limit orders'). The price dynamics is therefore the result of the interplay between the order book and the order flow. The study of the order book is very interesting both for academic and practical reasons. It provides intimate information on the processes of trading and price formation, and reveals a highly non trivial structure of the agents' expectations (see below): as such, it is of importance to test some basic notions of economics and models of market microstructure. The practical motivations are also clear. Regulatory bodies want to set the rules of exchange markets such as to produce fair, orderly trading that maximize flow. For market participants, issues such as the market impact or the relative merit of limit versus market orders are determined by the structure and dynamics of the order book.

Complete data on the order book of certain markets, such as the Paris Bourse (now Euronext), is now available, and contains a particularly abundant information. All orders (representing hundreds of thousands orders per month on liquid stocks) on all stocks are stored, which makes possible the reconstitution of the full order book at any instant of time, including orders that are not directly observable on traders' screens.

Many questions can be studied; the systematic investigation of these data sets is only very recent [9, 10, 11] and has motivated a number of interesting theoretical work [12, 13, 10, 14, 15, 16]. Here, we mostly focus on 'static' properties of the order book, such as the distribution of incoming limit orders, the average shape of the order book in the moving reference frame of the price, or the distribution of volume at the bid/ask. Many other 'dynamical' properties can also be analyzed, such as the response of the price to order flow, the full temporal correlation of the book, etc. In order to keep our study reasonably focused, we leave these dynamical aspects, that we have not fully understood yet, for a subsequent publication.

Our main results are as follows: (a) the price at which new limit orders are 
placed is, somewhat surprisingly, very broadly (power-law) distributed around the current bid/ask; (b) the average order book has a maximum away from the current bid/ask, and a tail reflecting the statistics of the incoming orders; (c) the distribution of volume at the bid (or ask) follows a Gamma distribution. Notably, we find exactly the same statistical features, for the three liquid stocks studied. We then study numerically a 'zero intelligence' model of order book which reproduces most of these empirical results. Finally, we show how the characteristic shape of the average order book can be analytically predicted, using a simple approximation.

The data provided by Paris Bourse gives the history of all transactions, with their price, volume and time stamp, of all quotes (bid and ask prices) with the corresponding volumes, and of all orders, with their price, volume and time stamp. This information allows in principle to reconstruct the whole order book at any instant of time. However, some limit orders (roughly 10\% of them) are modified or cancelled before being executed; unfortunately the data base only contains this qualitative information, but not the time at which a given order is modified/cancelled, nor the resulting new price. When reconstructing the order book at a given instant of time, we have therefore made two extreme assumptions, which lead to nearly identical conclusions. Either we discard these orders altogether, or we keep them until the time where we can be sure that they have been previously modified/cancelled, otherwise they would have been executed since the transaction price was observed to be below (for buy orders) or above (for sell orders) the corresponding limit price. The results given below uses the second procedure. We had available the data corresponding to all stocks during February 2001, from which we have extracted three of the most liquid stocks: France-Telecom (F.T.), Vivendi and Total. Some basic information is summarized in Table I for France-Telecom and Total. We expect that our findings will not depend on the particular month considered, but that some differences may appear when one studies stocks with smaller capitalisation (for example the average bid-ask spread is much larger): these questions will be investigated in the near future. Let us call $a(t)$ the ask price at time $t$ and $b(t)$ the level of bid price at time $t$. The midpoint $m(t)$ is the average between the bid and the ask: $m(t)=[a(t)+b(t)] / 2$. The smallest possible change of these quantities, called the 'tick', was 0.05 Euros for France-Telecom and Vivendi, and 0.10 Euros for Total.

We denote by $b(t)-\Delta$ the price of a new buy limit order, and $a(t)+\Delta$ the price of a new sell limit order. Notice that $\Delta$ can be negative (this is the only case where the spread $g(t)=a(t)-b(t)$ can be narrowed), but is always larger than $-g(t)$ (otherwise it would be a market order). A first interesting question concerns the distribution density of $\Delta$, i.e. the distance between the current price and the incoming limit order. We find that $P(\Delta)$ is identical for buy and sell orders (up to statistical fluctuations); the shape of $P(\Delta)$ is found to be very well 


\begin{tabular}{||l|c|c||}
\hline \hline Quantity & F.-T. & Total \\
\hline Initial/final price (Euros) & $90-65$ & $157-157$ \\
\hline Tick size (Euros) & 0.05 & 0.10 \\
\hline Total \# orders & 270,000 & 94,350 \\
\hline \# market orders & 28,600 & 9,300 \\
\hline \# trades & 176,000 & 60,000 \\
\hline Transaction volume & $75,610^{6}$ & $23,410^{6}$ \\
\hline Average bid-ask (ticks) & 2.0 & 1.4 \\
\hline Average volume at bid/ask & 2700 & 2400 \\
\hline \hline
\end{tabular}

Table 1: Some useful data for the studied stocks (February 2001). The transaction volume is in number of shares. The figures for Vivendi are nearly identical to those for France-Telecom.

fitted (see Fig. 1) by a power-law:

$$
P(\Delta) \propto \frac{\Delta_{0}^{\mu}}{\left(\Delta_{1}+\Delta\right)^{1+\mu}}, \quad \Delta \geq 1
$$

with an exponent $\mu \approx 0.6$ for all three stocks. This power-law extends from 1 tick to over 100 ticks (sometimes even 1000 ticks), corresponding to a relative change of price of $5 \%$ to $50 \%$ [17]. There are also orders placed at the bid (or ask) or within the spread. One finds that $P(\Delta=-1) \sim P(\Delta=0) \sim P(\Delta=1)$, and these orders add up to roughly half of the total number of orders. The distribution of orders Eq. (11) has a maximum around the current price (this was already noted in [9]). But the fact that $\mu<1$ means that the average $\Delta$ is formally infinite (although of course the distribution is ultimately cut-off for large $\Delta$ 's).

It is quite surprising to observe such a broad distribution of limit order prices, which tells us that the opinion of market participants about the price of the stock in a near future could be anything from its present value to $50 \%$ above or below this value, with all intermediate possibilities. This means that market participants believe that large jumps in the price of stocks are always possible, and place orders very far from the current price in order to take advantage of these large potential fluctuations. (Note that placing a limit order is free of charge.) A naive argument would then suggest that the probability to place an order at distance $\Delta$ should be proportional to the probability that the price moves more than $\Delta$ in order to meet the order. Since the tail of the distribution of price increments is a power-law with an exponent $\mu_{\delta p} \approx 3[4$, this would indeed lead to a power-law for $P(\Delta)$, but with a value $\mu=\mu_{\delta p}-1 \approx 2$ larger than the observed one. This however does not take into account the fact that market participants have different time horizons - large $\Delta$ 's presumably correspond to more patient investors. The value of $\mu$ should result from an interplay between the perceived distribution of future price changes and the distribution of time horizons, which 


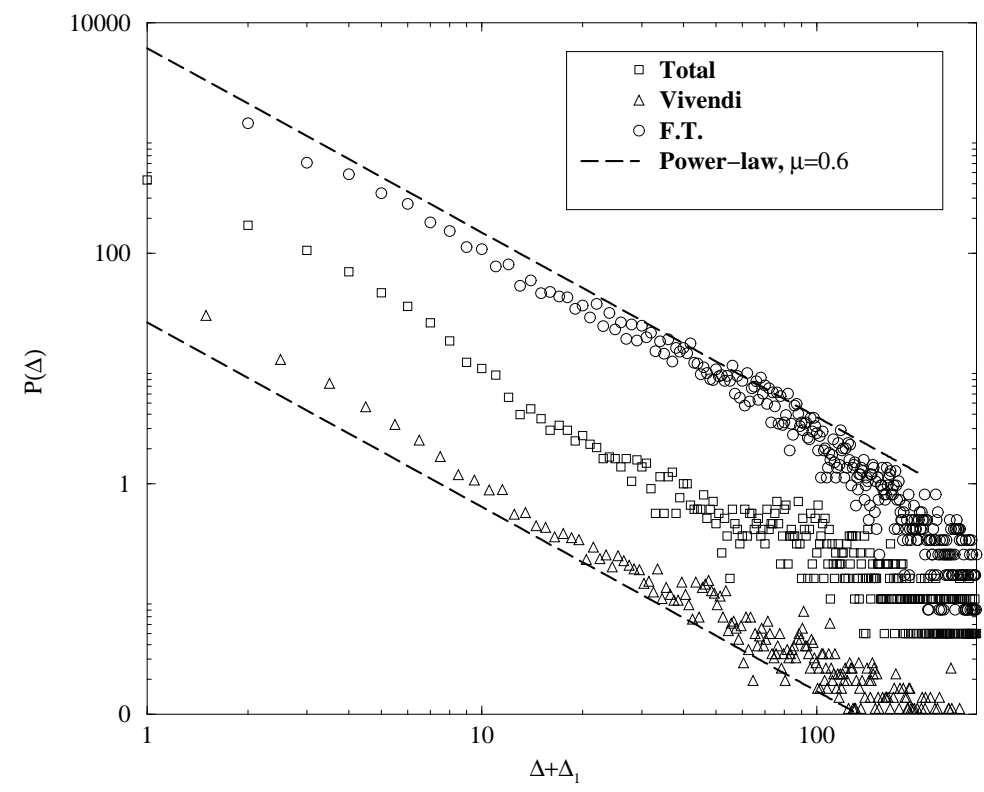

Figure 1: Number of incoming orders arriving at a distance $\Delta$ from the current best price, as a function of $\Delta_{1}+\Delta$ (in ticks), in log-log coordinates. We chose $\Delta_{1}=1$ for F.T., 0.5 for Vivendi and 0 for Total. We also found $P(\Delta=-1) \sim P(\Delta=0) \sim$ $P(\Delta=1)$ (not shown). The symbols correspond to buy orders, but sell orders show an identical distribution. The straight lines correspond to $\mu=0.6$. Note that the power-law crosses over to a faster decay beyond $\Delta \approx 100$. 
could well be itself a power-law. We feel that more theoretical work is needed to fully account for this striking result.

Limit orders strongly vary in volume. We find that the unconditional limit order size, $\phi$, is distributed uniformly in log-size, between 10 and 50,000 (both for buy or sell orders). One can study the correlation between the incoming volume $\phi$ (number of shares for a given order) and the distance $\Delta$ between the order and the current price. We find that the conditional averaged volume $\left.\langle\phi\rangle\right|_{\Delta}$ is roughly independent of $\Delta$ between 1 and 20 ticks, but decays as a power law $\Delta^{-\nu}$, with $\nu \sim 1.5$ beyond $\Delta^{*}$ (with $\Delta^{*} \approx 20$ ticks for $\mathrm{FT}$, and $\approx 50$ ticks for the other two stocks). Not unexpectedly, extremely far limit orders tend to be of smaller volumes. Since the average volume arriving at $\Delta$ is equal to the number of orders at $\Delta$ multiplied by $\left.\langle\phi\rangle\right|_{\Delta}$, one predicts, using the shape of $P(\Delta)$, that the distribution of incoming volume should then decay as $\Delta^{-1-\mu}$ for $\Delta<\Delta^{*}$ and as $\Delta^{-1-\mu-\nu}$ for large $\Delta>\Delta^{*}$, a feature that we have confirmed directly.

We now turn to the shape of the order book. The order flow is maximum around the current price, but an order very close to the current price has a larger probability of being executed and thus disappear from the book. It is thus not a priori clear what will be the average shape of the order book. Quite interestingly, we find that the (time-averaged) volume of the queue in the order book (that we will call for simplicity the 'average order book') is symmetrical, and has a maximum away from the current bid (ask): see Fig. 2. This was also noted in [9]. This shape furthermore appears to be universal, up to a rescaling of both the $\Delta$ axis and the volume axis, at least for the three stocks studied. In order to test the universality of this result, we are currently studying less liquid stocks, and in a different time interval. The empirical determination of the average order book is the central result of this paper, and simple models that explain this shape will be discussed below.

The next question concerns the volume fluctuations around this average shape. We first study the distribution $R(V)$ of volume at the bid (or ask). Again, the two are identical, and can be fitted by a Gamma distribution for the volume (Fig. $3)$ :

$$
R(V) \propto V^{\gamma-1} \exp \left(-\frac{V}{V_{0}}\right) .
$$

We find $\gamma \simeq 0.7-0.8$ for all three stocks. A Gamma distribution with $\gamma \leq 1$ has its maximum for $V=0$. This shows that the most probable value of the volume at bid is very small, although its typical value is quite large $\left(V_{0} \simeq 2700\right.$ for France-Telecom).

Therefore, the order book has strong fluctuations and at a given instant of time can look rather different from its average, Fig. 2. We have also studied the fluctuations of volume in the book as a function of $\Delta$, defined as:

$$
\sigma_{V}(\Delta)=\sqrt{\left.\left\langle V^{2}\right\rangle\right|_{\Delta}-\left.\langle V\rangle\right|_{\Delta} ^{2}} .
$$




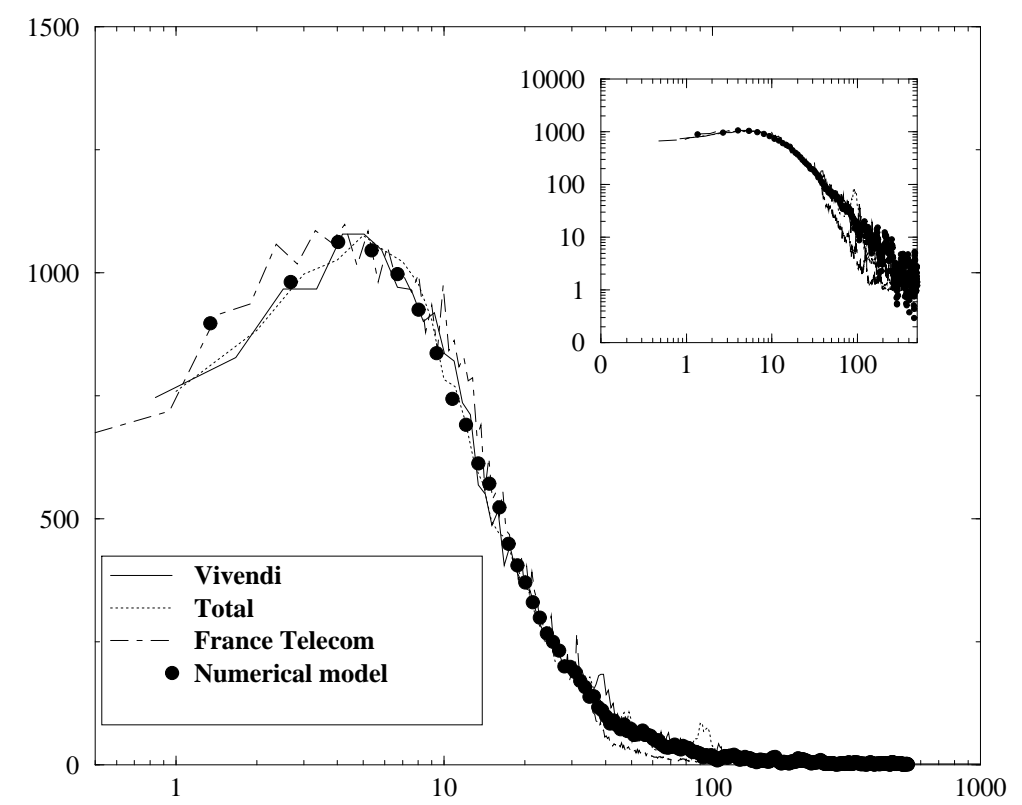

Figure 2: Average volume of the queue in the order book for the three stocks, as a function of the distance $\Delta$ from the current bid (or ask) in a log-linear scale. Both axis have been rescaled in order to collapse the curves corresponding to the three stocks. The thick dots correspond to the numerical model explained below, with $\Gamma=10^{-3}$ and $p_{m}=0.25$. Inset: same data in log-log coordinates.

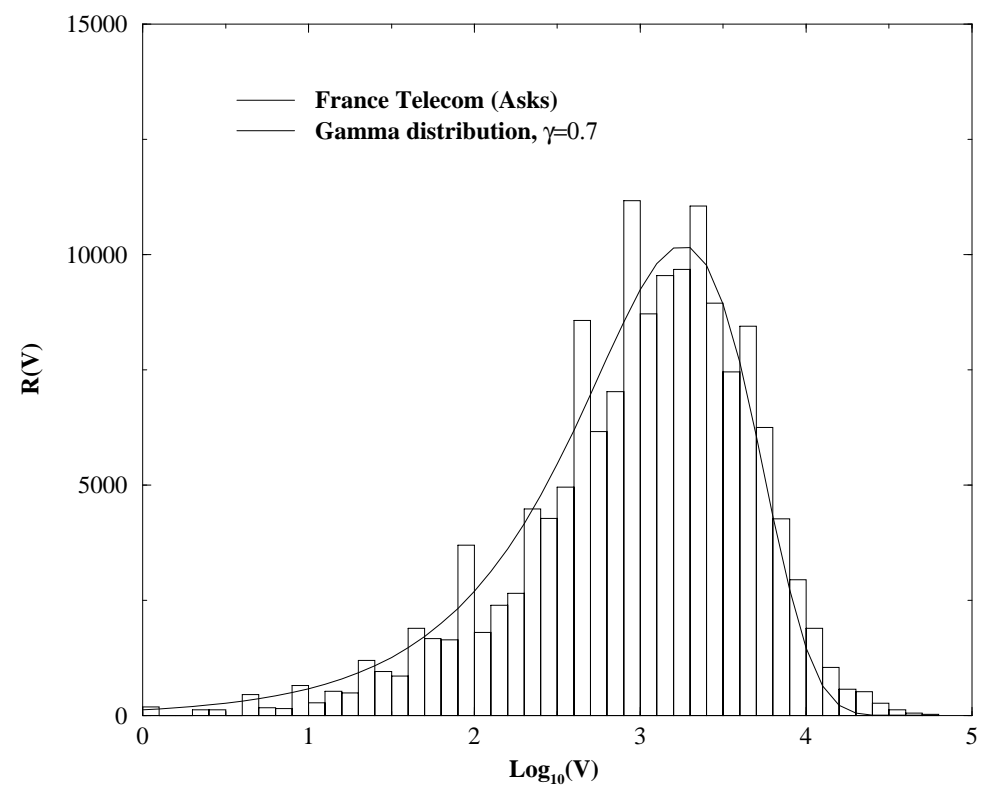

Figure 3: Histogram of the log-volume at the ask (same for bids), for France Telecom. The fit corresponds to Gamma distribution, Eq. (2) (after a change of variables to $\log V$ ), with $\gamma=0.7$. 
We find that $\sigma_{V}(\Delta)$ is of order 1 for $\Delta=1$ (as expected from the above Gamma distribution), and is roughly constant up to $\Delta=50$. This shows that far from the current price, the instantaneous order book shows large relative fluctuations, which is indeed reasonable. We have also studied the full covariance matrix of the fluctuations $C_{V}\left(\Delta, \Delta^{\prime}\right)$ (note that $C_{V}(\Delta, \Delta) \equiv \sigma_{V}^{2}(\Delta)$ ). For France-Telecom, its first eigenvalue corresponds to a dilation of the book, whereas the second one reflects an even/odd oscillation (orders are more numerous at prices in tenth of Euros than in twentieth of Euros). Higher eigenvalues are structureless, suggesting that the orderbook has no 'elasticity'. This is expected, since most investors do not have access to the order book in real time.

We now turn to a quantitative interpretation of the data. First, we have simulated an artificial 'zero intelligence' order book, much in the same spirit as in [11, 16], but more directly inspired from our empirical results. Limit orders, with a size distributed uniformly on a log scale, are launched at random with an a priori 'sprinkling' distribution $P(\Delta)$, which we take identical to the empirical one. Here we depart from [16], where all orders have unit size and the 'sprinkling' distribution is uniform between 0 and a certain $\Delta_{\max }$. The size distribution was actually found to play a minor role except in the far tail of the average order book, where the exponent $\nu$ introduced above becomes important. For example, we also studied the case of orders of equal size with very similar results.

On the other hand, choosing the correct 'rain' distribution $P(\Delta)$ is crucial to reproduce the shape of the order book. We also launch a certain fraction $p_{m}$ of market orders (chosen to be typically $1 / 6-1 / 4$ th of the total) in order to trigger trades. The results we find (in particular the averge size of the bid-ask spread) are quite sensitive to the value of $p_{m}$. Finally, with a constant probability $\Gamma$ (typically of order $10^{-3}$ ) per unit time, independently of both size and position in the book, an order is cancelled. We have found that this simple model is able to reproduce quantitatively many of the features observed in the empirical data, such a the shape of the order book (see Fig. 2), or the Gamma distribution of volume at the bid/ask $R(V)$, with a similar value for the exponent $\gamma$. This model also reproduces other empirical properties, such as the short time dynamics of the 'midpoint' and the sublinear volume dependence of the response to an incoming market order [18, 16, 19]. However, we note that the above simple model overestimates the average size of the queue close to $\Delta=0$. This might be due either to the fact that our procedure to treat modified orders leads to an systematic bias which is large near the best price, or that orders close to the best price have a non negligible strategic content that our model fails to capture.

Finally, we discuss a simple analytical approximation which allows us to compute the average order book from the ingredients of the numerical model. This was also attempted in [16]. Although very few details were given in [16], our method appears to be quite different from theirs. Consider sell orders. Those at distance $\Delta$ from the current ask at time $t$ are those which were placed there at a time $t^{\prime}<t$, and have survived until time $t$, that is, (i) have not been cancelled; 
(ii) have not been touched by the price at any intermediate time $t^{\prime \prime}$ between $t^{\prime}$ and $t$. An order at distance $\Delta$ at time $t$ in the reference frame of the ask $a(t)$ appeared in the order book at time $t^{\prime}$ at a distance $\Delta+a(t)-a\left(t^{\prime}\right)$. The average order book can thus be written, in the long time limit, as:

$$
\rho(\Delta, t)=\int_{-\infty}^{t} \mathrm{~d} t^{\prime} \int d u P(\Delta+u) \mathcal{P}\left(u \mid \mathcal{C}\left(t, t^{\prime}\right)\right) \mathrm{e}^{-\Gamma\left(t-t^{\prime}\right)},
$$

where $\mathcal{P}\left(u \mid \mathcal{C}\left(t, t^{\prime}\right)\right)$ is the conditional probability that the time evolution of the price produces a given value of the ask difference $u=a(t)-a\left(t^{\prime}\right)$, given the condition that the path always satisfies $\Delta+a(t)-a\left(t^{\prime \prime}\right) \geq 0$ at all intermediate times $t^{\prime \prime} \in\left[t^{\prime}, t\right]$. The evaluation of $\mathcal{P}$ requires the knowledge of the statistics of the price process. Because of the exponential cut-off $\mathrm{e}^{-\Gamma\left(t-t^{\prime}\right)}$, only the short time behaviour of the process is relevant where the confinement effects of the order book on the price are particularly important [10, 16, 19]. Nevertheless, to make progress, we will assume that the process is purely diffusive. In this case, $\mathcal{P}$ can be calculated using the method of images. One finds:

$$
\mathcal{P}\left(u \mid \mathcal{C}\left(t, t^{\prime}\right)\right)=\frac{1}{\sqrt{2 \pi D \tau}}\left[\exp \left(-\frac{u^{2}}{2 D \tau}\right)-\exp \left(-\frac{(2 \Delta+u)^{2}}{2 D \tau}\right)\right],
$$

where $\tau=t-t^{\prime}$ and $D$ is the diffusion constant of the price process.

After a simple computation, one finally finds, up to a multiplicative constant which only affects the overall normalisation of $\rho_{\mathrm{st}}(\Delta)=\rho(\Delta, t \rightarrow \infty)$ :

$$
\rho_{\mathrm{st}}(\Delta)=\mathrm{e}^{-\alpha \Delta} \int_{0}^{\Delta} \mathrm{d} u P(u) \sinh (\alpha u)+\sinh (\alpha \Delta) \int_{\Delta}^{\infty} \mathrm{d} u P(u) \mathrm{e}^{-\alpha u},
$$

where $\alpha^{-1}=\sqrt{D / 2 \Gamma}$ measures the typical variation of price during the lifetime of an order, and fixes the scale over which the order book varies. When the distribution of order flows $P(\Delta)$ has the power-law shape Eq. (II) with $\mu<1$, the parameter $\alpha$ can be rescaled away in the 'continuous' limit where $\alpha^{-1}$ is much larger than the tick size (which is the relevant limit for stocks, where $\alpha^{-1} \sim 10$ ). In this case, the shape of the average order book only depends on $\mu$ and $\hat{\Delta}=\alpha \Delta$, and is given by the following convergent integral:

$$
\rho_{\mathrm{st}}(\hat{\Delta})=\mathrm{e}^{-\hat{\Delta}} \int_{0}^{\hat{\Delta}} \mathrm{d} u u^{-1-\mu} \sinh (u)+\sinh (\hat{\Delta}) \int_{\hat{\Delta}}^{\infty} \mathrm{d} u u^{-1-\mu} \mathrm{e}^{-u} .
$$

For $\Delta \rightarrow 0$, the average order book vanishes in a singular way, as $\rho_{\mathrm{st}}(\Delta) \propto$ $\Delta^{1-\mu}$, whereas for $\Delta \rightarrow \infty$, the average order book reflects the incoming flow of orders: $\rho_{\text {st }}(\Delta) \propto \Delta^{-1-\mu}$. We have plotted in Fig. (1 the average order book obtained numerically from the 'zero intelligence' model (which is very close to empirical data, see Fig. 2) and compared it with Eq. (7), with $\mu=0.6$ and various choices of parameters. After rescaling the two axes, the various numerical 


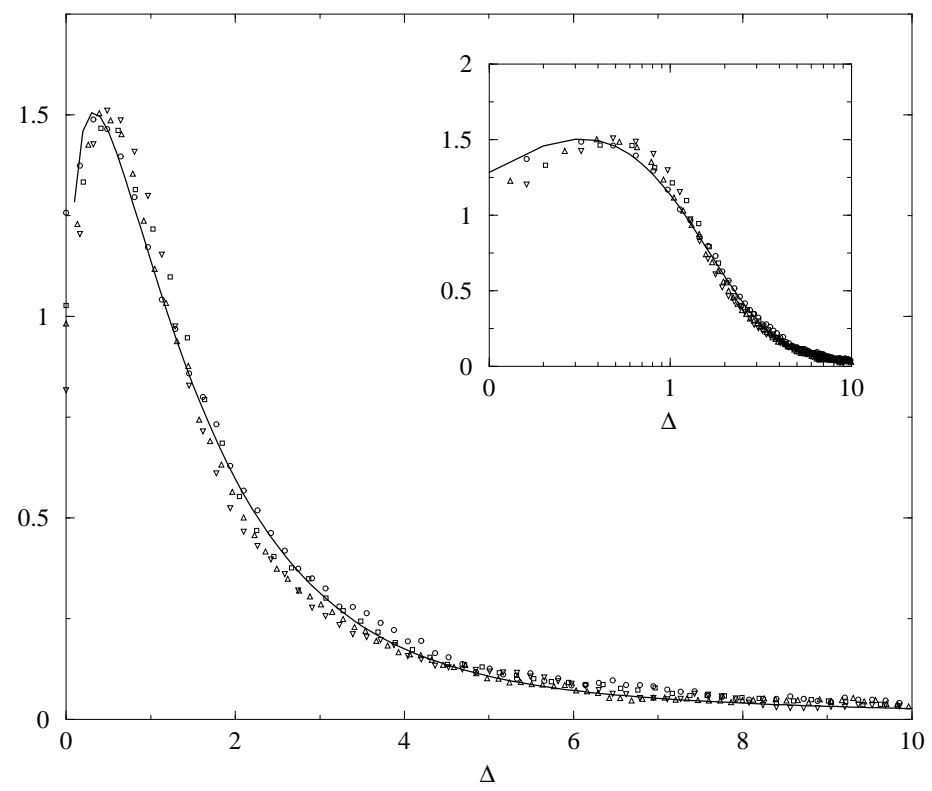

Figure 4: The average order book of the numerical model with various choices of parameters $\left(\mu=.6, p_{m} \in\{1 / 4,1 / 6\}\right.$, and $\Gamma \in\left\{10^{-3}, 510^{-4}\right\}$ is compared to the approximate analytical prediction, (full curve), Eq. (7). After rescaling the axes, the various results roughly scale on the same curve, which is well reproduced by our simple analytic argument. The inset shows the same data in a log-linear representation. 
models lead to very similar average order books, and the analytic approximation appears rather satisfactory, bearing in mind the roughness of our 'diffusive' approximation.

The shape of the average order book therefore reflects the competition between a power-law flow of limit orders with a finite lifetime, and the price dynamics that removes the orders close to the current price. These effects lead to a universal shape which will presumably hold for many different markets, provided the lifetime of orders is sufficiently long compared to the typical time between trades, and the volatility on the scale of the orders lifetime is somewhat larger than the ticksize. I On the other hand, the detailed shape of the book in the immediate vicinity of the best price (i.e., around $\Delta=0$ ) is not expected to be universal.

In conclusion, we have investigated several 'static' properties of the order book. We have found that the results appear to be independent of the stock studied. The most interesting features concern (i) the statistics of incoming limit order prices, which follows a power-law around the current price with a diverging mean - suggesting, quite surprisingly, that market participants believe that very large variations of the price are possible within a rather short time horizon; and (ii) the shape of the average order book, which can be quantitatively reproduced using a 'zero intelligence' numerical model, and qualitatively predicted using a simple approximation. One of the most interesting open problems is, in our view, to explain the clear power-law behaviour of the incoming orders and the difference between our results and those of [17]. The dynamical properties of the order book, and the way the price reacts to order flow, will be the subject of a further study 19.

Acknowledgements: We thank Jean-Pierre Aguilar, Jelle Boersma, Damien Challet, J. Doyne Farmer, Laurent Laloux, Andrew Matacz, Philip Seager and Denis Ullmo for stimulating and useful discussions.

\section{References}

[1] for a recent review, see: R. Cont, Quantitative Finance, 1, 223 (2001), and refs. therein.

[2] J.-P. Bouchaud and M. Potters, Théorie des Risques Financiers, Aléa-Saclay, 1997; Theory of Financial Risks, Cambridge University Press, 2000.

[3] R. Mantegna \& H. E. Stanley, An Introduction to Econophysics, Cambridge University Press, 1999.

\footnotetext{
${ }^{1}$ Preliminary results indeed show that the average order book in futures markets has a similar hump away from the current midpoint.
} 
[4] V. Plerou, P. Gopikrishnan, L.A. Amaral, M. Meyer, H.E. Stanley, Phys. Rev. E60 6519 (1999).

[5] T. Lux, Applied Financial Economics, 6, 463, (1996).

[6] D. M. Guillaume, M. M. Dacorogna, R. D. Davé, U. A. Müller, R. B. Olsen and O. V. Pictet, Finance and Stochastics 195 (1997).

[7] for a review, see e.g. J.-F. Muzy, J. Delour, E. Bacry, Eur. Phys. J. B 17, 537-548 (2000), and refs. therein.

[8] F. Longin, Journal of Business, 69383 (1996)

[9] B. Biais, P. Hilton, C. Spatt, An empirical analysis of the limit order book and the order flow in the Paris Bourse, Journal of Finance, 50, 1655 (1995)

[10] S. Maslov, Simple model of a limit order-driven market, Physica A 278, 571 (2000); S. Maslov, M. Millis, Price fluctuations from the order book perspective - empirical facts and a simple model, Physica A 299, 234 (2001)

[11] D. Challet, R. Stinchcombe, Analyzing and modelling $1+1 d$ markets, Physica A 300, 285 (2001)

[12] P. Bak, M. Paczuski, and M. Shubik, Physica A 246, 430 (1997)

[13] David L.C. Chan, David Eliezer, Ian I. Kogan, Numerical analysis of the Minimal and Two-Liquid models of the Market Microstructure, preprint cond-mat/0101474

[14] H. Luckock, A statistical model of a limit order market, Sidney University preprint (September 2001).

[15] F. Slanina, Mean-field approximation for a limit order driven market model, preprint cond-mat/0104547

[16] Marcus G. Daniels, J. Doyne Farmer, Giulia Iori, Eric Smith, How storing supply and demand affects price diffusion, preprint cond-mat/0112422.

[17] Such a broad distribution, with very far away limit orders, was also found for British stocks, J.D. Farmer, private communication and I. Zovko, J. D. Farmer, preprint cond-mat/0206280. Note that these authors find $\mu \approx 1.5$.

[18] P. Gopikrishnan, V. Plerou, X. Gabaix, H.E. Stanley, Statistical Properties of Share volume traded in Financial Markets, Phys. Rev E 62, R4493 (2000).

[19] J.P. Bouchaud, M. Mézard, M. Potters, in preparation. 